\title{
Across the Universe
}

\author{
Highly sophisticated materials properties and engineering efforts are crucial for the success of missions for space \\ exploration and settlement.
}

t's the year 2083, and almost 11 billion people live on an overcrowded Earth. We struggle to keep up with the demand for natural resources. Platinum and gold prices have hit the roof. There is not enough energy to power our world. Water is a luxury.

Is this the future? Certainly a dim version of it, and not the only reason we have turned our heads towards the sky. Humans imagined space exploration before potential concerns about resource shortage and reduced living space on Earth developed, even before the technology to enable it came to be. And it remains one of the most extraordinary human endeavours. In this issue, we explore some of the aspects of materials properties required for space missions.

In a Comment, Tommaso Ghidini ${ }^{1}$, head of the Structures, Mechanisms and Materials Division of the European Space Agency (ESA) guides us through the extreme properties that materials used for space missions need to possess. During their trip from Earth, to orbit and then their return to Earth or another planet, spacecrafts face extreme temperatures (hundreds of ${ }^{\circ} \mathrm{C}$, positive or negative), solar and cosmic radiation, and atmospheric dynamic pressure. Materials tests are of course held on Earth but also on the International Space Station (ISS), a manned facility that is in low Earth orbit. The US, Russian, Japanese, European and Canadian space agencies use the ISS as a testbed for materials used mostly for missions to the Moon and Mars.

Additive manufacturing plays a significant part in space missions as it allows for the quick fabrication of small amounts of customized parts. Currently, polymers can be $3 \mathrm{D}$ printed in the ISS, and metals will follow soon. The bigger goal is of course making the missions more sustainable: the ability to recycle and repair equipment in space will not only improve their lifetime but also reduce the global amount of material used. ESA has also recently shown that planet soil can be used as the primary material in 3D printing equipment by using focused sunlight ${ }^{2}$, making the development of on-planet manufacturing units a plausible and fascinating reality.

Space exploration entails even more ambitious projects. Breakthrough Starshot (part of the Breakthrough Initiatives (https:// breakthroughinitiatives.org), funded by billionaire Yuri Milner) is definitely one of them; its aim is to send an unmanned

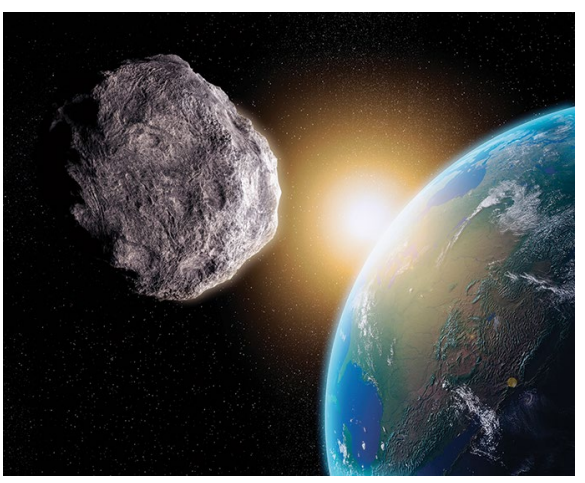

Credit: Science Photo Library/Alamy Stock Photo

nanospacecraft to explore our closest neighbouring star system, Proxima Centauri, and then transmit photos and other relevant data from its travels, back to Earth. Proxima Centauri is 4.2 light years away, so reaching it within a couple of decades would require very high speeds. The key to achieving such levels of speed is a lightsail, a reflective surface that can be propelled by intense laser beams. Solar sailing may sound futuristic to many, but it was implemented in JAXA's IKAROS spacecraft $^{3}$ in 2010, while the Planetary Society plans to send their second lightsail spacecraft in orbit around Earth later this year ${ }^{4}$. However, both lightsails were launched aboard rockets, while the maximum speed IKAROS reached was only $400 \mathrm{~m} \mathrm{~s}^{-1}$; this is about five orders of magnitude lower than the $0.2 c$ speed that the Starshot lightsail is looking to reach.

Clearly, several questions must be addressed before the spacecraft sails away. Calculations so far suggest that the energy capacity needed would be of the order of $10 \mathrm{GW} \mathrm{m}^{-2}$. A phased laser array has been put forth as the most accommodating source, while the ideal shape of the lightsail is spherical ${ }^{5}$. In a Perspective in this issue, Harry Atwater and colleagues ${ }^{6}$ from Caltech take a closer look at the material properties that the lightsail should possess to attain high reflectivity, large bandwidth and low mass necessary for the spacecraft to reach such speeds. Other challenges include the interaction of the lightsail with interstellar dust as well as building a small but powerful enough transmitter to send data back to Earth; travelling at the speed of light, we will still have to wait an additional handful of years for the first images of the Proxima Centauri neighbourhood to arrive on Earth.
But looking for a new home is not the only purpose of these space missions. Space mining has been proposed as a possible additional supply of noble and rare-earth metals on Earth. In fact, asteroids are rich in materials such as platinum, nickel, iron, tungsten, nitrogen, hydrogen and even water. A study from the Keck Institute for Space Studies (KISS) at Caltech back in $2012^{7}$ concluded that mining near-Earth asteroids weighing around 500-1,000 tonnes is actually feasible and should rely on a closed ecosystem of investigating and locating potential targets, bringing them into lunar orbit with a powerful solar electric propulsion system, and then exploiting them in manned space stations in lunar orbit. This industry is of course at its very early stages, but the United States and Luxembourg ${ }^{8}$ have already developed the legal framework that allows private companies to exploit space resources; Luxembourg has also announced an additional investment of $€ 200$ million for asteroid mining companies ${ }^{9}$. It is unclear whether exploiting these resources to develop technology on Earth will be economically viable, but chasing asteroids could be instrumental in sustaining human life in space, as well powering space missions, especially when combined with in situ manufacturing facilities.

Surely to many, these endeavours may seem more appropriate for a science-fiction movie rather than a scientific journal. And we probably are still decades away from casual space travel. But there is no doubt that as technology progresses, space will come closer. Shooting for the stars now is the right thing to do.

Published online: 24 September 2018 https://doi.org/10.1038/s41563-018-0193-3

\footnotetext{
References

1. Ghidini, T. Nat. Mater. https://doi.org/10.1038/s41563-018-0184-4 (2018).

2. ESA https://go.nature.com/2MVjmrf (2017).

3. JAXA http://global.jaxa.jp/projects/sat/ikaros/ (2015).

4. Planetary Society http://www.planetary.org/explore/projects/ lightsail-solar-sailing/ (2018).

5. Popova, H., Efendiev, M. \& Gabitov, I. Math. Meth. Appl. Sci. 40, 1346-1354 (2016)

6. Atwater, H. A. et al. Nat. Mater. https://doi.org/10.1038/s41563018-0075-8 (2018)

7. Brophy, J. et al. Asteroid Retrieval Feasibility Study (Caltech, 2012); http://kiss.caltech.edu/final_reports/Asteroid_final_report.pdf

8. Ram, A. Financial Times https://www.ft.com/content/af15f0e4 707a-11e7-93ff-99f383b09ff9 (19 October 2017).

9. Space Resources https://spaceresources.public.lu/en.html (2018).
} 